日韓セッション

\title{
Endoscopic sinus surgery (ESS)
}

\author{
Motohiko Suzuki \\ Department of Otorhinolaryngology, Nagoya City University
}

Endoscopic sinus surgery (ESS) has been recently paid attention, because endoscopic approach provides excellent magnification and visualization. ESS is also minimally invasive surgery. ESS is now standard procedure for surgery of chronic rhinosinusitis. However, there is a possibility of minor and severe complications. ESS may induce intracranial injury, orbital injury, optic nerve injury, and nasolacrimal duct injury, resulting in cerebral spinal fluid leak, meningitis, double vision, vision damage, epiphora, eye discharge, and so on.
Surgical field visualization is important for good outcomes and no severe complications. It is very difficult to find landmarks in the poor surgical visualization by massive bleeding in the surgical field. Considering this, control of bleeding during surgery is important.

Recent reports also have showed the efficacy and safety of the endoscopic approach in management of various neoplasms.

I will give a presentation about ESS in the present and future. 